\title{
Merkel cell carcinoma: Our experience in this rare pathology
}

\author{
Pablo Rosado ${ }^{1}$, Luis Junquera ${ }^{2}$, Blanca Vivanco ${ }^{3}$, Luis García-Consuegra ${ }^{4}$, Lorena Gallego ${ }^{4}$ \\ ${ }^{1}$ Atending, Department of Oral and Maxillofacial Surgery. Central University Hospital, Oviedo, Spain \\ ${ }^{2}$ Professor of Oral and Maxillofacial Surgery, University of Oviedo Dental School and Central University Hospital, Oviedo, \\ Spain \\ ${ }^{3}$ Department of Pathologic Anatomy. Central University Hospital, Oviedo, Spain \\ ${ }^{4}$ Staff surgeon. Department of Oral and Maxillofacial Surgery. Cabueñes Hospital, Gijón, Spain
}

Correspondence:

Asturias Central University Hospital,

Celestino Villamil St,

33009 Oviedo, Spain,

pablorosado80@hotmail.com

Received: $21 / 04 / 2010$

Accepted: 26/08/2010

Rosado P, Junquera L, Vivanco B, García-Consuegra L, Gallego L. Merkel cell carcinoma: Our experience in this rare pathology. Med Oral Patol Oral Cir Bucal. 2011 Sep 1;16 (6):e736-9.

http://www.medicinaoral.com/medoralfree01/v16i6/medoralv16i6p736.pdf

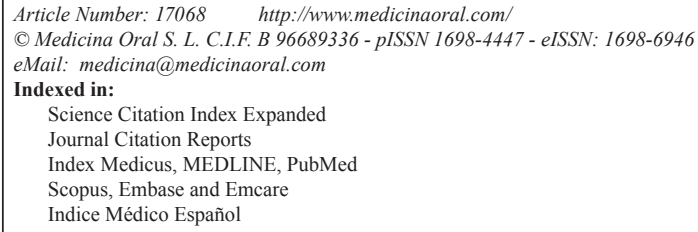

\begin{abstract}
Merkel cell carcinoma (MCC) was first described in 1972 by Toker, who described five cases of the so-called "trabecular carcinoma of the skin". MCC is a rare, aggressive skin cancer that affects mainly the elderly. Sun exposed areas are mainly affected, specially the head and neck. Immunohistochemical analysis is essential to reach a correct diagnosis. According to the origin of MCC, the tumor expresses both epithelial and neuroendocrine markers. MCC has a propensity for recurrence, regional and distant metastases. Several treatment options are available, such as surgical excision or Moh's surgery, accompanied by neck dissection or radio and chemotherapy in advanced cases. The present study aims to evaluate the clinical behaviour and the evolution of five cases of this disease that were treated at our institution (reference to 1100000 inhabitants) in the last five years. It is stressed on the bad prognosis of this pathology, which presents high rates of locoregional recurrence, even though the recent advances in radio and chemotherapy.
\end{abstract}

Key words: Merkel cell carcinoma, head and neck neoplasms, spontaneous regression, skin surgery.

\section{Introduction}

Friedrich Sigmund Merkel first described in 1875 touch cells in the skin of pigs that nowadays are known to act as mechanoreceptors. These Merkel cells (MC) are also involved in different embryological processes such as the formation of subepidermal nerve plexus, eccrine sweat glands and hair follicles. MC may derive from epidermal cells or from neural crest cells that migrate to epidermis during embryogenesis.

Merkel cell carcinoma (MCC) was first described in
1972 by Toker (1), who described five cases of the socalled "trabecular carcinoma of the skin", because of the typical architecture of the tumor. MCC is a rare, aggressive skin cancer with an estimated age-adjusted incidence of 0.24 per 100,000 person-years (2) that affects mainly the elderly.

Clinical appearance is often non-specific, occurring usually as painless intradermal erythematous lesions, with a progressive tendency to ulceration as it grows (Fig. 1). 
Pathogenetic factors such as ultraviolet radiation may contribute to tumor development. Sun-damaged skins are mostly affected, specially the head and neck region (3). MCC has been also associated with immunosuppression and the development of other synchronous or metachronous tumors (4).

The present study aims to evaluate the clinical behaviour and the evolution of five cases of this disease that were treated at our institution (reference to 1100000 inhabitants) in the last five years.

\section{Cases Reports \\ Case 1}

This case has been published in 2005 (5). A 79-year-old woman presented to our department with a 1-month rapidly enlarging $3 \mathrm{~cm}$ tumor on her right cheek. A biopsy of the tumor revealed a definite histopathologic diagnosis of MCC of intermediate subtype. Immunohistochemical staining showed positive reactions for antibodies against cytokeratin 20, neuronspecific enolase, chromogranin, $\gamma$-enolase, and synaptophysin. Further image studies revealed no local, regional, or distant metastatic spread of the disease. Patient was classified initially as stage II of the Memorial Sloan-Kettering Cancer Center staging system.

Twenty days after the biopsy, the tumor decreased to almost half its size, and after 49 days it was no longer visible macroscopically. Three months after the biopsy a 1 $\mathrm{cm}$ residual nodule was removed. The excised specimen showed no evidence of tumor cells. After 60 months follow up period, complete clinical and histological regression of MCC was observed.

\section{Case 2}

A 72-year-old man presented to his private dermatologist with a red mass located in the subnasal region that was diagnosed histopathologically as MCC of intermediate subtype. The patient was sent to our department and underwent the complete removal of the primary lesion and bilateral functional neck dissection (Fig. 1). Closure was achieved with local flaps and the treatment was complemented with radiotherapy. Nine months after surgery the patient developed a large left cervical metastasis and a left $4 \mathrm{~cm}$ parascapular mass. A radical neck dissection involving the lesion was done and the parascapular lesion was also removed with margins. Histopathological analysis confirmed the diagnosis of distant metastases of MCC. According to stage IV, a chemotherapeutic regimen was given (carboplatin and etoposide phosphate), which has been maintained until now. The patient is free of disease after 54 months since the diagnosis.

Case 3

A 52 year-old man presented to our department with a $1.5 \mathrm{~cm}$ cutaneous lesion affecting the left submental region, accompanied with a ipsilateral submental node.

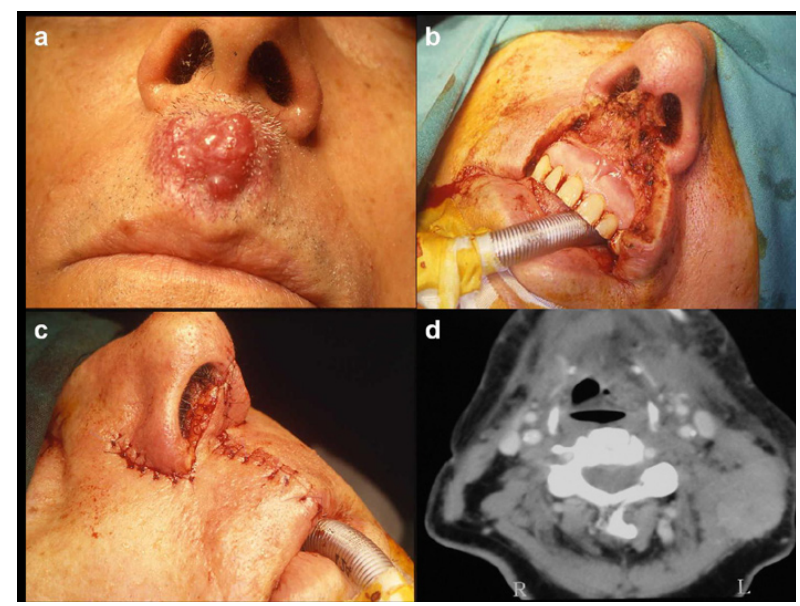

Fig. 1. Case 2. (a) Patient showed a rapidly growing, non-ulcerated, red nodule on subnasal region skin. (b) Complete removal of the lesion with adequate margins. (c) Closure of the defect with local flaps. (d) CT scan showing local dissemination (left neck)

The diagnosis of MCC with ganglionar dissemination was obtained with a biopsy and a fine needle aspiration (stage III). Then a complete removal of the lesion and a functional ipsilateral neck dissection were performed. Treatment was complemented with postoperative radiotherapy. One year after the surgery, the patient died because of a pancreatic carcinoma, but without signs of locoregional recidive.

\section{Case 4}

A 74 year-old man presented to his physician with a 2 $\mathrm{cm}$ mass in the left temporal region. A complete excision was performed under general anesthesia and the diagnosis of MCC was achieved. One month later, both left cervical and parotid masses appeared. A fine needle aspiration biopsy confirmed the diagnosis of regional metastases of the primary tumor (stage III) and so a functional neck dissection including the masses and a parotidectomy were done. The treatment was complemented postoperatively with radiotherapy. The patient is still alive after 36 months after surgery and free of disease.

\section{Case 5}

An 81 year-old-woman presented to our Department with a red, hard $2 \mathrm{~cm}$ mass located in the right check (Fig. 2). An incisional biopsy confirmed the diagnosis of MCC. Further extension studies confirmed no local, regional, or distant metastatic spread of MCC (stage I). The treatment consisted in wide excision and ipsilateral supra-omohyoid neck dissection. The closure was achieved with the combination of a rhomboidal and a Bichat fat flap. The patient is alive and free of disease after 24 months of follow-up. 


\section{Discussion}

Merkel cell carcinoma is a very aggressive primary cutaneous neoplasm. From a histological point of view, MCC is included in a set of tumors known as "small round blue cell neoplasms" (6). Pathological analysis reveals the presence of small blue cells with sparse cytoplasm and medium-sized nuclei, accompanied of frequent mitoses and expansion to subcutaneous tissue (Fig. 3).

Following histological criteria, MCC can be divided into three subtypes. The trabecular subtype presents cells arranged in distinctly organoid clusters and trabeculae with occasional ribbons. The intermediate subtype, the most frequent and aggressive, exhibits a solid and diffuse growth pattern with less compacted cells. The small cell types shows irregular, hyperchromatic cells with scant cytoplasm and malignant cells that are arranged in linear patterns infiltrating stromal structures. These tumors mimic in form and behavior those of small cells of the lung. All cases presented the intermediate subtype.

Immunohistochemical analysis is essential to reach a correct diagnosis. According to the origin of $\mathrm{MCC}$, the tumor expresses both epithelial and neuroendocrine markers. Epithelial differentiation is characterized by the expression of low-molecular-weight cytokeratins, specially Cytokeratin 20 (CK-20) (Fig. 4a). MCC is also characteristically negative for Thyroid Transcription Factor 1 (TTF-1), which is expressed in epithelial cells of the thyroid, lung and brain (Fig. 4b). These markers are used simultaneously for the differential diagnosis of $\operatorname{MCC}(7)$.

Neuroendocrine markers are also expressed by MCC (Fig. $4 \mathrm{c}-\mathrm{e})$, which can include neuron-specific enolase (NSE), CD56, Chromogranin A (CrA) and synaptophysin. Ki-67 index is also characteristically high (Fig. 4f).

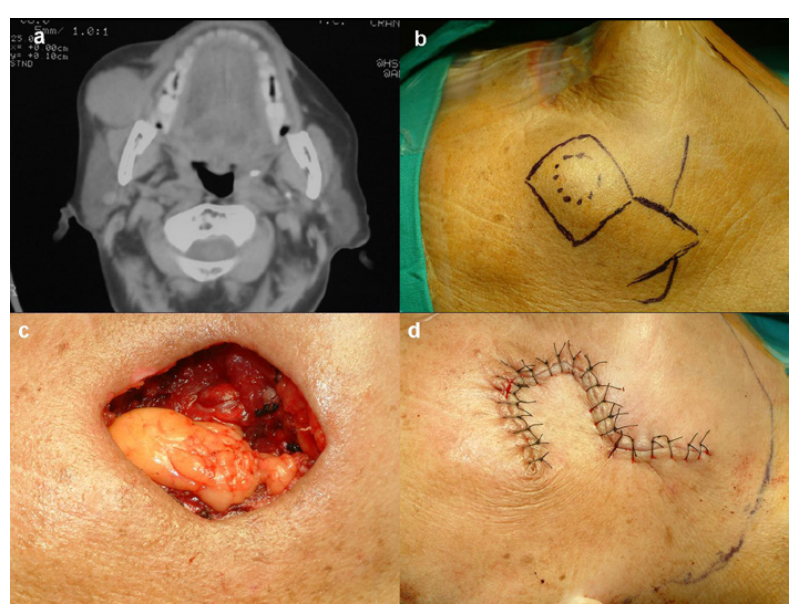

Fig. 2. Case 5. (a) CT scan showing a $2 \mathrm{~cm}$ subcutaneous mass in the right cheek. (b) Preoperative design of removal and reconstruction. (c) Wide extent of the defect with the Bichat flat pad prepared to close the intraoral defect. (d) External closure with a rhomboidal flap.

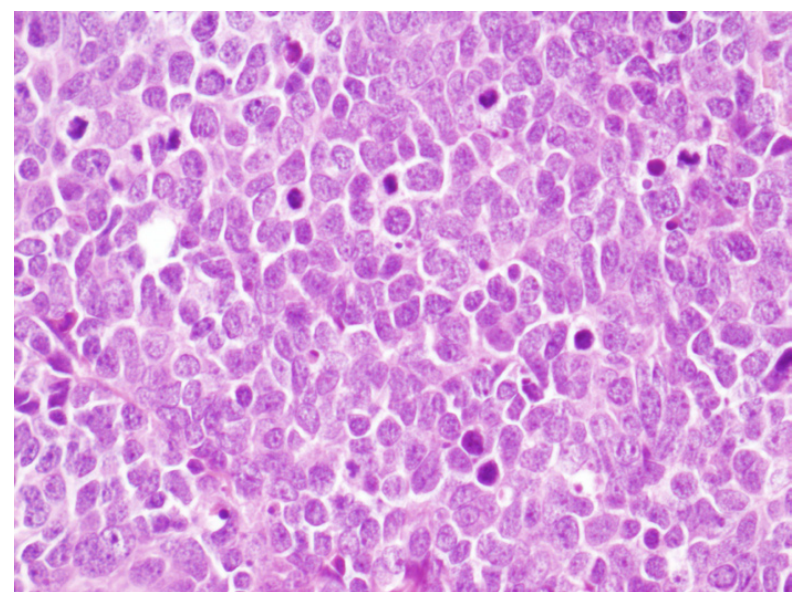

Fig. 3. Case 3. Small blue cells are characterized by sparse cytoplasm and medium-sized nuclei, accompanied of frequent mitoses (hematoxilin-eosin, x40).

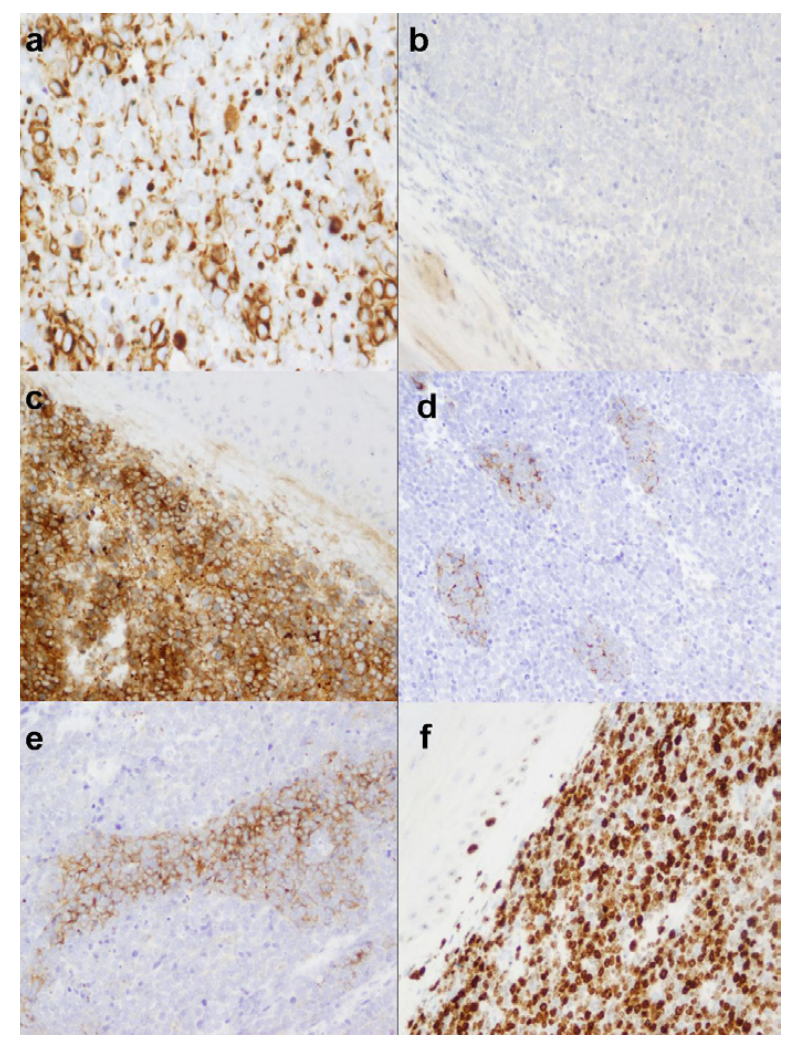

Fig. 4. Case 4 (a) Punctate or dot-like perinuclear staining with antibody to cytokeratin $20(40 \mathrm{x})$. (b) Negative staining for thyroid transcription factor allows differential diagnosis with other small cells neoplasms (20x). (c) Positive immunostaining for neuron-specific enolase (x20). (d) Positive immunostaining for chromogranin (20x). (e) Positive immunostaining for synaptophysin (20x). (f) Ki67 index shows positivity higher than $90 \%$. 
Memorial Sloan-Kettering Cancer Center group has proposed a staging system (8) based on clinical parameters. This classifies the patients as follows: stage I localized disease with a primary tumor dimension $<2$ $\mathrm{cm}$, and stage II if primary tumor dimension is $>2 \mathrm{~cm}$. Patients with regional or distant metastatic disease are classified as stages III and IV, respectively.

$\mathrm{MCC}$ has a propensity for recurrence, regional and distant metastases. Several treatment options are available. Complete excisions with wide margins or Moh's surgery have been recommended for treatment of primary lesions in order to achieve free margins. In addition, prophylactic lymphadenectomy has been also recommended as lymph node metastases develop in approximately $50 \%$ of patients (9). Recently, sentinel lymph node has been described as a new weapon, providing information about prognosis and identifying the draining lymph node pattern for surgery and adjuvant radiation therapy (RT).

$\mathrm{MCC}$ is also a radiosensitive tumor. In accordance to this, patients treated with surgery alone compared with those treated with surgery and RT have increased rates of locoregional recurrence (10). Several chemotherapeutic regimens have been used in patients with metastatic MCC. These include combination therapies with cisplatin, doxorubicin and vincristine or with etoposide and platinum, with initial overall response rates of approximately $60 \%$ (11). Despite this fact, the median overall survival of patients with metastatic disease is 10 months. All our cases presented initial response to treatment and only one died one year after treatment because of a pancreatic carcinoma. So overall survival mean in our study was 37.2 months. In we take in account the only patient with metastatic disease, the survival after beginning with the chemotherapy was 54 months, noticeably higher than the expected.

Although this is a rare phenomenon, there is also the possibility of spontaneous regression of MCC (12), as we have previously reported. However, the mechanisms of this feature remain unclear, apoptotic events have been reported to play important roles in its pathogenesis.

\section{References}

1. Toker C. Trabecular carcinoma of the skin. Arch Dermatol. 1972 ;105:107-10.

2. Agelli M, Clegg LX. Epidemiology of primary Merkel cell carcinoma in the United States. J Am Acad Dermatol. 2003;49:832-41.

3. Gass JK, Chan SK, Rytina E, Greenberg DC, Burrows NP. Multiple primary malignancies in patients with Merkel cell carcinoma. J Eur Acad Dermatol Venereol. 2010;24:601-3.

4. Luaces Rey R, Fernández Alba J, Martín R, García Rozado A, Paradela S, Robles O, et al. Merkel cell carcinoma of the head and neck: report of seven cases. Med Oral Patol Oral Cir Bucal. 2008;13:E390-4.

5. Junquera L, Torre A, Vicente JC, García-Consuegra L, Fresno MF. Complete spontaneous regression of Merkel cell carcinoma. Ann Otol Rhinol Laryngol. 2005;114:376-80.
6. Pisick E, Skarin AT, Salgia R. Recent advances in the molecular biology, diagnosis and novel therapies for various small blue cell tumors. Anticancer Res. 2003;23:3379-96.

7. Hanly AJ, Elgart GW, Jorda M, Smith J, Nadji M. Analysis of thyroid transcription factor-1 and cytokeratin 20 separates merkel cell carcinoma from small cell carcinoma of lung. J Cutan Pathol. 2000;27:118-20.

8. Allen PJ, Bowne WB, Jaques DP, Brennan MF, Busam K, Coit DG. Merkel cell carcinoma: prognosis and treatment of patients from a single institution. J Clin Oncol. 2005;23:2300-9.

9. Lawenda BD, Thiringer JK, Foss RD, Johnstone PA. Merkel cell carcinoma arising in the head and neck: optimizing therapy. Am J Clin Oncol. 2001;24:35-42.

10. Gillenwater AM, Hessel AC, Morrison WH, Burgess M, Silva EG, Roberts D, et al. Merkel cell carcinoma of the head and neck: effect of surgical excision and radiation on recurrence and survival. Arch Otolaryngol Head Neck Surg. 2001;127:149-54.

11. Bichakjian CK, Lowe L, Lao CD, Sandler HM, Bradford CR, Johnson TM, et al. Merkel cell carcinoma: critical review with guidelines for multidisciplinary management. Cancer. 2007;110:1-12.

12. Junquera L, Torre A, Gallego L. Re: 'Complete spontaneous regression in Merkel cell carcinoma’. J Plast Reconstr Aesthet Surg. 2009;62:262. 